\title{
Defluviicoccus vanus glycogen accumulating organisms (DvGAOs) are less competitive than polyphosphate accumulating organisms (PAOs) at high temperature
}

Li Wang, ${ }^{\mathrm{a}, \mathrm{b}}$ Adrian Oehmen, ${ }^{\mathrm{c}}$ Chencheng Le, ${ }^{\mathrm{a}}$ Jianbo Liu, ${ }^{\mathrm{a}, \mathrm{d}}$ and Yan Zhou ${ }^{\mathrm{a}, \mathrm{e},{ }^{*}}$

a Advanced Environmental Biotechnology Centre, Nanyang Environment and Water Research Institute, Nanyang Technological University, 1 Cleantech Loop, Singapore,

637141, Singapore

b Interdisciplinary Graduate School, Nanyang Technological University, Singapore 639798, Singapore

c School of Chemical Engineering, The University of Queensland, St. Lucia, Queensland 4072, Australia

${ }^{\mathrm{d}}$ College of Environmental Science and Engineering, Hunan University, Changsha, 410082, China

e School of Civil and Environmental Engineering, Nanyang Technological University, Singapore 639798, Singapore

*Corresponding author: Yan Zhou

Address: 50 Nanyang Avenue, Singapore 639798

E-mail: zhouyan@ntu.edu.sg

Tel: $(+65) 67906103$ 


\section{Supplementary material}
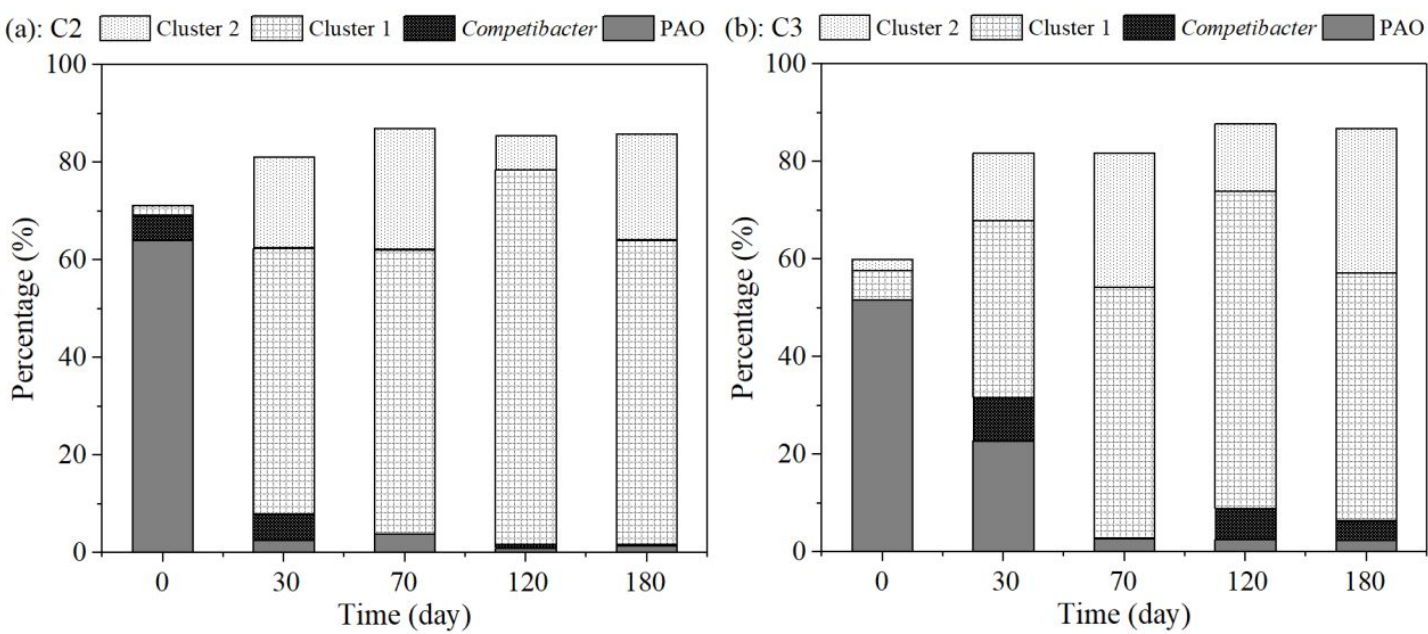

Figure S1 The relative abundance of bacterial transition in C2-GAO (a) and C3-GAO

(b) reactors. 


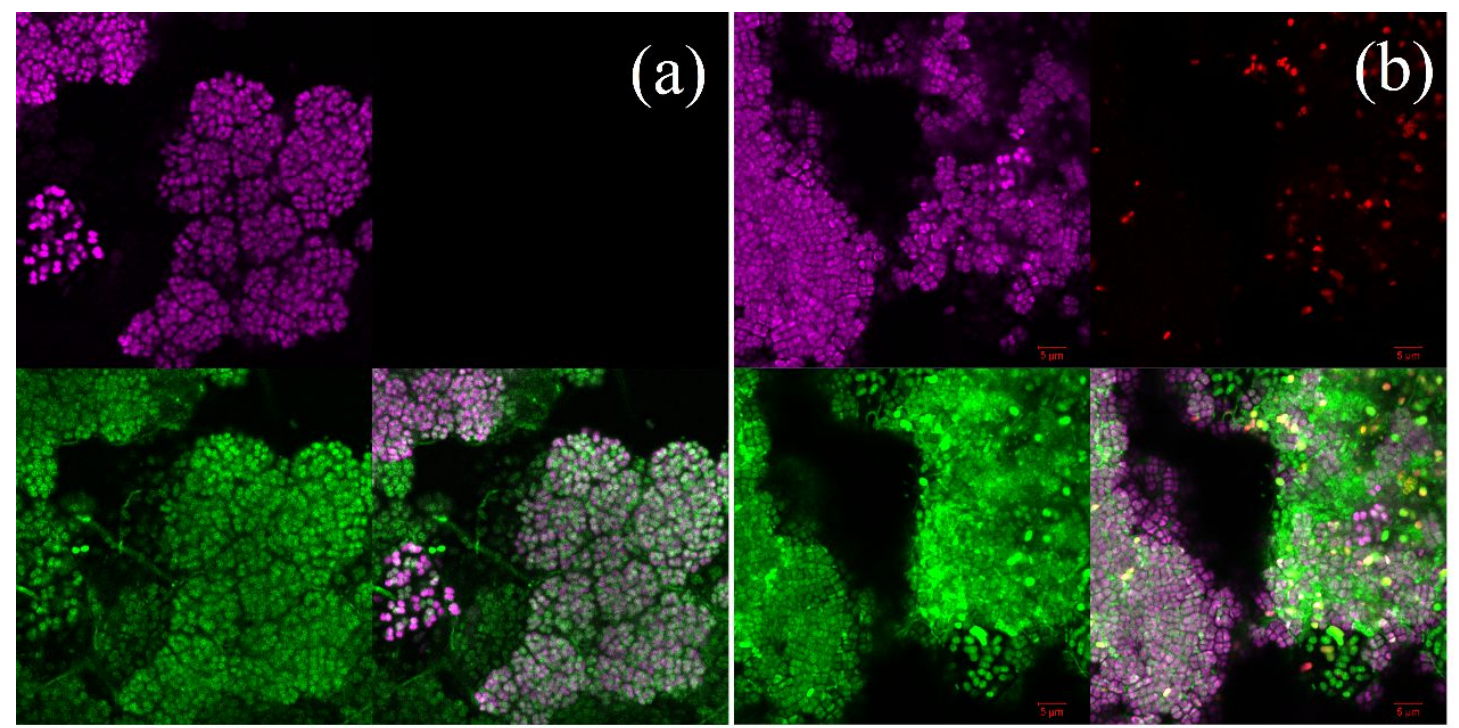

Figure S2 Bacterial population distribution in C2-GAO (a) and C3-GAO (b) by applying fluorescence in situ hybridization (purple: glycogen accumulating organisms, red: polyphosphate accumulating organisms and green: all bacterial). 
Table S1. The individual VFA uptake rate of mixed carbon source (C-mmol/g VSS /h) in $\mathrm{C} 2-\mathrm{GAO}$ and $\mathrm{C} 3-\mathrm{GAO}$.

\begin{tabular}{llc}
\hline Standards & Concentration equation & $\mathrm{R}^{2 *}$ \\
3-hydroxypropaonic acid (3HP) & $\mathrm{y}=2 \mathrm{E}+8 \mathrm{x}-2 \mathrm{E}+6$ & 0.9982 \\
3-hydroxybutyric acid (3HB) & $\mathrm{y}=9 \mathrm{E}+7 \mathrm{x}+6 \mathrm{E}+6$ & 0.9995 \\
3-hydroxypentanoic acid (3HV) & $\mathrm{y}=9 \mathrm{E}+7 \mathrm{x}-6 \mathrm{E}+5$ & 0.9999 \\
3-hydroxyhexanoic acid (3HHx) & $\mathrm{y}=1 \mathrm{E}+8 \mathrm{x}+4 \mathrm{E}+6$ & 0.9996 \\
3-hydroxyoctanoic acid (3HO) & $\mathrm{y}=1 \mathrm{E}+8 \mathrm{x}+3 \mathrm{E}+6$ & 0.9998 \\
3-hydroxydecanoic acid (3HD) & $\mathrm{y}=1 \mathrm{E}+8 \mathrm{x}+5 \mathrm{E}+6$ & 0.9998 \\
3-hydroxydodecanoic acid (3HDOD) & $\mathrm{y}=1 \mathrm{E}+8 \mathrm{x}+4 \mathrm{E}+6$ & 0.9997 \\
3-hydroxytetradecanoic acid (3HTRD) & $\mathrm{y}=7 \mathrm{E}+7 \mathrm{x}-2 \mathrm{E}+5$ & 0.9993 \\
3-hydroxyhexadecanoic acid (3HTED) & $\mathrm{y}=5 \mathrm{E}+7 \mathrm{x}+8 \mathrm{E}+5$ & 0.9999 \\
\hline
\end{tabular}

*Correlation coefficient 
Table S2. The total VFA uptake rate of mixed carbon source (C-mmol/g VSS /h) in C2-GAO and C3-GAO.

\begin{tabular}{lllll}
\hline Substrate & Acetate-GAO & Propionate-GAO & Acetate-PAO & Propionate-PAO \\
\hline $\mathrm{C} 2+\mathrm{C} 3$ & $6.39 \pm 0.01$ & $4.14 \pm 0.08$ & $8.90 \pm 0.12$ & $9.21 \pm 0.18$ \\
$\mathrm{C} 2+\mathrm{C} 3+\mathrm{C} 4+\mathrm{C} 5$ & 5.22 & $2.80 \pm 0.01$ & 4.51 & 5.25 \\
$\mathrm{C} 2+\mathrm{C} 3+\mathrm{ISO}-\mathrm{C} 4+\mathrm{ISO}-\mathrm{C} 5$ & 4.65 & $2.80 \pm 0.43$ & 4.09 & 4.88 \\
\hline
\end{tabular}


Table S3. The synthesized new PHA to VFA (C-mol/ C-mol) in C2-GAO and C3-GAO.

\begin{tabular}{lllllll}
\hline Substrate & \multicolumn{3}{c}{ Acetate-GAO } & \multicolumn{3}{c}{ Propionate-GAO } \\
& PH4MV & PHHx & PH2MHx & PH4MV & PHHx & PH2MHx \\
C4 & 0.26 & 0.37 & 0.19 & 0.35 & 0.57 & 0.29 \\
ISO-C4 & 0.42 & 0.08 & 0.02 & 0.78 & 0.23 & 0.09 \\
C2+C3+C4+C5 & 0.02 & 0.11 & 0 & 0.07 & 0.18 & 0 \\
C2+C3+ISO-C4+ISO-C5 & 0.06 & 0 & 0 & 0.21 & 0 & 0 \\
\hline
\end{tabular}

